\title{
Geometry-Assisted Topological Transitions in Spin Interferometry
}

\author{
M. Wang, ${ }^{1}$ H. Saarikoski®,${ }^{2}$ A. A. Reynoso, ${ }^{3}$ J. P. Baltanás, ${ }^{4}$ D. Frustaglia ${ }^{4,5,{ }^{*}}$ and J. Nitta ${ }^{1,6,7, \dagger}$ \\ ${ }^{1}$ Department of Materials Science, Tohoku University, Sendai 980-8579, Japan \\ ${ }^{2}$ RIKEN Center for Emergent Matter Science (CEMS), Wako Saitama 351-0198, Japan \\ ${ }^{3}$ INN-CONICET, Centro Atómico Bariloche, 8400 Bariloche, Argentina \\ ${ }^{4}$ Departamento de Física Aplicada II, Universidad de Sevilla, E-41012 Sevilla, Spain \\ ${ }^{5}$ Freiburg Institute for Advanced Studies (FRIAS), Albert-Ludwigs Universität Freiburg, D-79104 Freiburg, Germany \\ ${ }^{6}$ Center for Spintronics Research Network, Tohoku University, Sendai 980-8577, Japan \\ ${ }^{7}$ Organization for Advanced Studies, Center for Science and Innovation in Spintronics (Core Research Cluster), \\ Tohoku University, Sendai 980-8577, Japan
}

(Received 26 July 2019; revised manuscript received 5 November 2019; published 30 December 2019)

We identify a series of topological transitions occurring in electronic spin transport when manipulating spin-guiding fields controlled by the geometric shape of mesoscopic interferometers. They manifest as distinct inversions of the interference pattern in quantum conductance experiments. We establish that Rashba square loops develop weak-(anti)localization transitions (absent in other geometries as Rashba ring loops) as an in-plane Zeeman field is applied. These transitions, boosted by nonadiabatic spin scattering, prove to have a topological interpretation in terms of winding numbers characterizing the structure of spin modes in the Bloch sphere.

DOI: 10.1103/PhysRevLett.123.266804

Spin-orbit (SO) interaction emerges as a vector potential leading to momentum-dependent magnetic textures that govern the evolution of itinerant spins in mesoscopic systems [1]. The resulting spin dynamics can exhibit a series of geometric and topological properties of significant interest as the Aharonov-Casher (AC) effect [2], the electromagnetic dual of the Aharonov-Bohm (AB) effect [3]. Ring-shaped Rashba SO interferometers develop radial magnetic textures that can be manipulated by applying additional in-plane Zeeman fields [4]. At a critical point, the hybrid Rashba-Zeeman field undergoes a topological transition from a rotating texture (dominated by the radial Rashba component) to an oscillating one (dominated by the uniform Zeeman component) leaving an imprint on the spin dynamics with remarkable consequences in quantum transport experiments. This was first discussed by LyandaGeller [5] in the limit of adiabatic spin transport, where the fields are sufficiently strong to force the itinerant spins to stay locally aligned with the Rashba-Zeeman field texture during transport [6]. In this limit, field and spin textures share identical topological features captured by the itinerant spins in the form of a geometric Berry phase [7] that manifests as a spin-dependent magnetic flux in the quantum interference of spin carriers (recalling the $A B$ effect [8-15]) that can be switched on or off by the in-plane Zeeman field [16]. We have shown that topological spin phase transitions are still possible in ring-shaped interferometers away from the adiabatic limit [17]: The transition is determined by the field's topology through an effective Berry phase related to the winding number of the spin states around the poles of the Bloch sphere. Yet, the experimental realization is challenging since the required in-plane Zeeman fields are strong, resulting in spin-induced dephasing [18] that ruins spin interference as the transition point is approached [4].

Recently, spin reorientation by geometric means has been considered after noticing that out-of-plane spin components develop in curved Rashba SO channels $[19,20]$. An early proposal was discussed in polygonal interferometers [21-22], where the vertices act as spinscattering centers that hinder the emergence of $\mathrm{AC}$ and geometric phases. This suggests that sharp curvatures could soften the stiffness of Rashba-driven spin textures under the action of in-plane Zeeman fields, turning topological spin phase transitions possible at much weaker field strengths.

Here, we report transport simulations and experiments in square-shaped Rashba spin interferometers. Our simulations show that the topological properties of the spin states undergo a transition as an in-plane Zeeman field is applied. The topological transitions manifest as a sign reversal of spin interference leading to a distinctive weak-(anti)localization pattern for weak Zeeman fields that contrasts with the case of ring-shaped interferometers. We demonstrate this response in a transport experiment with semiconductor square-shaped spin interferometers, establishing the existence of spin-texture topological transitions assisted by the polygonal geometry of the SO channels.

One-dimensional (1D) transport simulations.-1D Rashba SO wires along a generic direction $\hat{\gamma}$ in the $x y$ plane are modeled by the Hamiltonian [21] 


$$
H_{\gamma}=\frac{p_{\gamma}^{2}}{2 m^{*}}+\frac{\alpha}{\hbar} p_{\gamma}(\hat{\gamma} \times \hat{z}) \cdot \sigma,
$$

with $p_{\gamma}$ the linear momentum of the spin carriers, $\alpha$ the Rashba SO strength, and $\sigma$ the Pauli matrix vector. The second term in Eq. (1) appears as an effective in-plane magnetic field $\boldsymbol{B}_{\mathrm{SO}}=\left(2 \alpha / \hbar g \mu_{B}\right) p_{\gamma}(\hat{\gamma} \times \hat{z})$ coupled to the itinerant spins, with $\mu_{B}$ the Bohr magneton and $g$ the $g$-factor. Notice that $\boldsymbol{B}_{\mathrm{SO}}$ is perpendicular to $\hat{\gamma}$ and inverts its sign for counterpropagating carriers due to the factor $p_{\gamma}$. Polygonal Rashba loops with $N$ sides and perimeter $P$ can be built by arranging segments of length $l=P / N$ modeled by Eq. (1), leading to effective-field discontinuities at the vertices with a significant effect on spin dynamics. Ring-shaped loops are described by taking the limit $N \gg 1$ with constant $P$, where a radial effective field emerges and the discontinuities disappear [23] (see Fig. S2 in the Supplemental Material [24]). Moreover, we introduce an in-plane uniform magnetic field $\boldsymbol{B}_{Z}$ along the $x$ axis that interacts with the itinerant spins through a Zeeman-coupling term $\left(g \mu_{B} / 2\right) B_{Z} \sigma_{x}$ to be added in Eq. (1). The Rashba SO and Zeeman coupling strengths are quantified in terms of spin precession angles during propagation along the loop's perimeter, $k_{\mathrm{SO}} P$ and $k_{Z} P$, with $k_{\mathrm{SO}}=\left(\alpha m^{*} / \hbar^{2}\right), k_{Z}=\left(g \mu_{B} B_{Z} m^{*} / 2 \hbar^{2} k_{F}\right)$, and $k_{F}$ the Fermi wave number. The hybrid Rashba-Zeeman field texture changes topology at the turning point $k_{Z} P=k_{\mathrm{SO}} P$.

We calculate the zero-temperature quantum conductance of 1D ring- and square-shaped loops with source and drain contact leads along the $x$ axis by applying the LandauerBüttiker formalism, which identifies conductance with transmission. Moreover, we apply a semiclassical approach (valid when the system's size $P$ is much larger than the Fermi wavelength $\lambda_{F}$, in coincidence with our experimental conditions) where the quantum transmission results from the interference of classical paths contributing to the transport of carriers through the loop. Along their way, spin carriers collect different quantum phases according to their particular paths, spin species, and fields experienced. In experiments where disorder and/or sample average is relevant, only pairs of paths with the same geometric length contribute significantly to quantum interference (contributions from other paths simply average out). These correspond to time-reversal (TR) orbital paths starting and ending at the source contact. In the absence of fields, TR paths produce constructive interference that reinforces backscattering leading to magnetoconductance minima, something referred to as weak localization (WL). The introduction of SO fields can revert this effect by means of spin AC phases contributing to destructive TR-path interference, producing magnetoconductance maxima known as weak antilocalization (WAL). Large Zeeman fields can restore WL by "freezing" the spin dynamics [18]. Hence, WL/WAL signals in the presence of SO and Zeeman fields reflect the response of the spin degree of freedom [24].

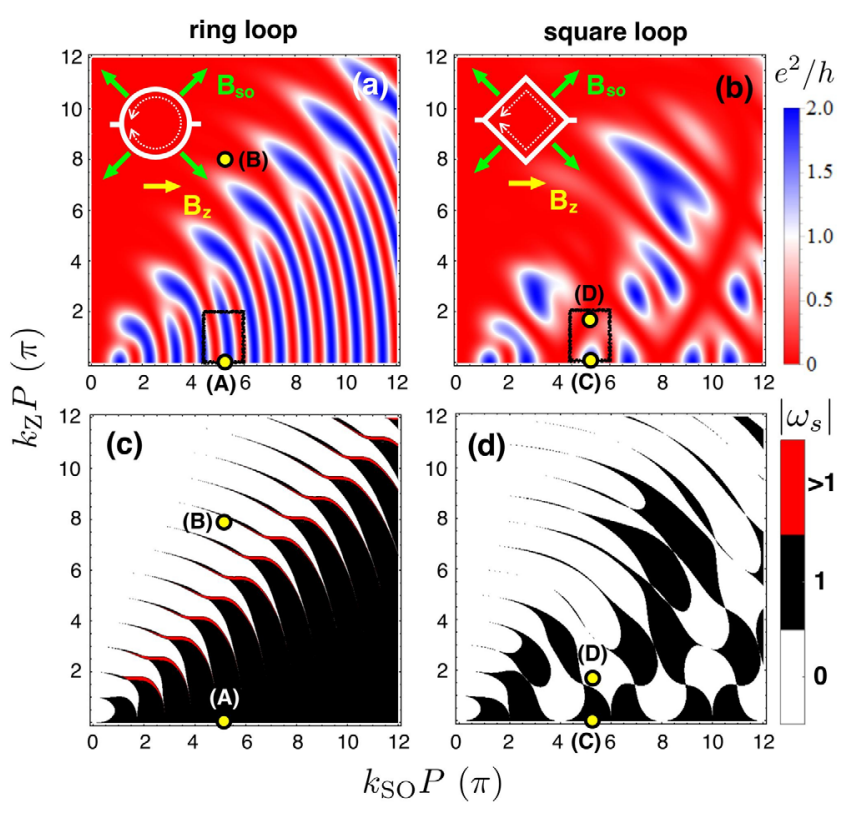

FIG. 1. Linear conductance of ring (a) and square (b) loops by TR-path interference (red: WL; blue: WAL) and winding number of spin textures $\left|\omega_{s}\right|$ in ring (c) and square (b) loops as a function of the Rashba SO and in-plane Zeeman field strengths. Field and spin textures for points (A), (B), (C), and (D) are shown in Fig. 2. For dominating Rashba strengths (field winding $\left|\omega_{B}\right|=1$ ), conductance maxima (blue) take place only when $\left|\omega_{s}\right|=1$ (dark). Checkerboardlike patterns in (b) and (d) are indicative of spin-texture topological transitions for weak Zeeman fields in squares, in contrast to rings. Rashba SO field textures (insets) correspond to counterclockwise itinerant spins. Interference fringes in (a) and (b) disappear for dominating Zeeman fields due to a frozen spin dynamics. The rectangles in (a) and (b) show the approximate zones accessible by experiments. Red zones in (c) $\left(\left|\omega_{s}\right|>1\right)$ are due to resonances discussed in Refs. [17] and [26].

Figures 1(a) and 1(b) show the conductance of 1D ring- and square-shaped loops calculated from TR-path interference at the lowest order, i.e., by considering only single-winding (counter)clockwise paths (see insets, dotted arrows), as a function of $k_{\mathrm{SO}} P$ and $k_{Z} P$. The contrast found between the interference patterns of Figs. 1(a) and 1(b) indicates that spin phases $\phi_{s}$ contributing to WAL (blue zones) develop quite differently in rings and squares. Spin phases are dominated by the expectation value of the spin Hamiltonian $H_{s}=\left(g \mu_{B} / 2\right)\left[\boldsymbol{B}_{\mathrm{SO}}(\ell)+\boldsymbol{B}_{Z}\right] \cdot \boldsymbol{\sigma}$ over the itinerant-spin eigenstates $\left|\chi_{s}(\ell)\right\rangle, \phi_{s} \sim \int_{0}^{P}\left\langle\chi_{s}(\ell)\left|H_{s}\right| \chi_{s}(\ell)\right\rangle d \ell$, i.e., by the projection of the spin texture $\hat{\boldsymbol{s}}(\ell)=$ $\left\langle\chi_{s}(\ell)|\boldsymbol{\sigma}| \chi_{s}(\ell)\right\rangle$ over the field texture $\boldsymbol{B}(\ell)=\boldsymbol{B}_{\mathrm{SO}}(\ell)+\boldsymbol{B}_{Z}$, where $\ell$ parametrizes the loop's perimeter. Moreover, spin and field textures can be characterized topologically in terms of (integer) winding numbers around the $z$ axis defined as $\omega_{s / B}=(1 / 2 \pi) \int_{0}^{P} d \ell\left[\hat{\boldsymbol{n}}_{s / B} \times\left(d \hat{\boldsymbol{n}}_{s / B} / d \ell\right)\right] \cdot \hat{\boldsymbol{z}}$, with $\hat{\boldsymbol{n}}_{s / B}(\ell)$ the normalized $x y$ projections of $\hat{\boldsymbol{s}}(\ell)$ and $\boldsymbol{B}(\ell)$, respectively, where $\omega_{s / B}=0, \pm 1$ in the cases studied here [29]. WAL 


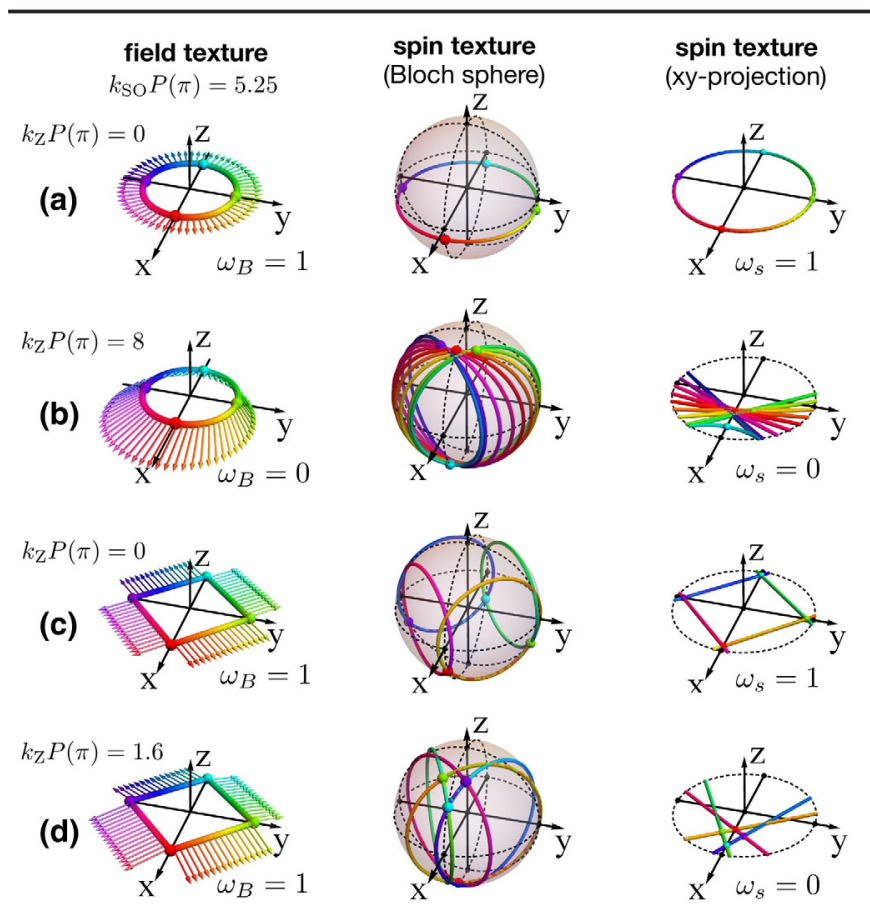

FIG. 2. Field and spin textures for hybrid Rashba-Zeeman ring and square loops for points (A), (B), (C), and (D) shown in Fig. 1. Field and spin topologies are defined by winding numbers $\omega_{B}$ and $\omega_{s}$ around the origin in the $x y$ plane. For rings (a) and (b) a change in $\omega_{s}$ requires large in-plane Zeeman fields changing $\omega_{B}$ as well. For squares (c) and (d) weak Zeeman fields are sufficient to change $\omega_{s}$ without changing $\omega_{B}$, instead. The Rashba SO components of the field textures correspond to counterclockwise itinerant spins. The color code refers to the position along the loops perimeter.

arises only when the spin phases are sufficiently large (otherwise, WL prevails). Figures 1 and 2 show that this happens when the field and spin textures share the same topology $\left(\omega_{s}=\omega_{B}\right)$, i.e., when the local projection $\hat{\boldsymbol{s}}(\ell)$. $\boldsymbol{B}(\ell)$ has a definite sign all over the loop's perimeter. We elaborate on this in the following paragraphs.

In Rashba rings, spin and field textures are well correlated even in the presence of Zeeman fields (away from the critical point $\left.k_{Z} P=k_{\mathrm{SO}} P\right)[4,17,30,31]$. This is illustrated in Fig. 1(c), where we plot the spin winding number $\left|\omega_{s}\right|$. For field textures dominated by the radial SO component $\left(k_{Z} P<k_{\mathrm{SO}} P,\left|\omega_{B}\right|=1\right)$ the spin textures develop a finite $\omega_{s}$ (black zone) corresponding to circular trajectories described by the tip of $\hat{\boldsymbol{s}}(\ell)$ winding around the $z$ axis in the Bloch sphere, see Fig. 2(a). As for the conductance, Fig. 1(a), it develops regular AC interference bands with a period of $\pi$ in $k_{\mathrm{SO}} P$ units. In the adiabatic limit $\left(k_{\mathrm{SO}} P / \pi\right) \gg 1$, spin and field textures eventually coincide within the ring's plane. Moreover, the $\mathrm{AC}$ interference bands bend as the Zeeman field is introduced. This shift has been experimentally observed and explained as a spin geometric phase effect [4]. Figure 1(c) shows that changing the spin winding $\omega_{s}$ (white zone) requires large Zeeman fields beyond the critical point $\left(k_{Z} P>k_{\mathrm{SO}} P\right)$ that changes the field winding $\omega_{B}$ as well, see Fig. 2(b).

In Rashba squares, Fig. 1(b), we find that the AC oscillation period doubles to $2 \pi$ in $k_{\mathrm{SO}} P$ units with respect to rings, in agreement with previous works for Rashba squares without Zeeman fields [21]. The discontinuity of the Rashba SO field at the corners turns adiabatic spin evolution impossible even for large $k_{\mathrm{SO}} P$, hindering the development of AC phases. Spin eigenstates stay away from the loop's plane, forming complex spin textures sensitive to Zeeman-field perturbations-see Figs. 2(c) and 2(d). The checkerboardlike pattern emerging in Fig. 1(b) as $k_{Z} P$ increases shows interference fringes fully reversed by a weak Zeeman field on a scale $\pi$ in $k_{Z} P$ units. This patterncontrasting with the interference bands of Fig. 1(a) for rings-is due to topological changes in the spin texture. Indeed, Fig. 1(d) shows that the spin winding $\omega_{s}$ is fully correlated with the interference pattern of Fig. 1(b): Conductance maxima (WAL) in Fig. 1(b) (blue zones) are the consequence of large spin phases that can only be produced by spin textures with finite windings - and significant projection on the equally winding field texture, see Fig. 2(c)—appearing as dark zones in Fig. 1(d). A weak Zeeman field introduces major distortions on the highly nonadiabatic spin textures, producing a decorrelation between spin- and field-texture windings - see Fig. 2(d) that impede the gathering of spin phases contributing to WAL, therefore restoring WL. This demonstrates that spintexture topological transitions are accessible in square loops by using weak Zeeman fields that do not modify the topology of the field texture, in contrast to what is observed in rings [24].

Experiment and discussion.-Our experimental setup consists of an array of Rashba square-shaped spin interferometers, which we use to investigate the topological transition induced by an in-plane Zeeman field. This configuration allows for a clear observation of the spin interference thanks to the ensemble averaging naturally provided by the sample.

We employed an InGaAs quantum well (QW) epitaxially grown on an InP (001) substrate [24]. The carrier density dependence of the Rashba SO parameter is obtained from the analysis of the beating patterns appearing in the gate voltage dependence of Shubnikov-de Haas oscillations. The Rashba SO parameter $\alpha$ is electrically controlled from $\alpha=-1.17 \mathrm{peVm}$ at $N_{s}=2.0 \times 10^{12} \mathrm{~cm}^{-2}$ $\left(V_{g}=-2 \mathrm{~V}\right)$ to $\alpha=-3.62 \mathrm{peVm} \quad$ at $N_{s}=1.26 \times$ $10^{12} \mathrm{~cm}^{-2}\left(V_{g}=-5 \mathrm{~V}\right)$.

A top-gate-attached $40 \times 40$ square loop array with square side length $l=700 \mathrm{~nm}$ was fabricated by electron beam lithography and reactive ion etching. A laser scanning microscope image is shown in Fig. 3(a). The square loop array was covered with a 200-nm thick $\mathrm{Al}_{2} \mathrm{O}_{3}$ insulator made by atomic layer deposition and a $\mathrm{Cr} / \mathrm{Au}$ top gate electrode in order to tune $\alpha[32,33]$. All the measurements 
(a)
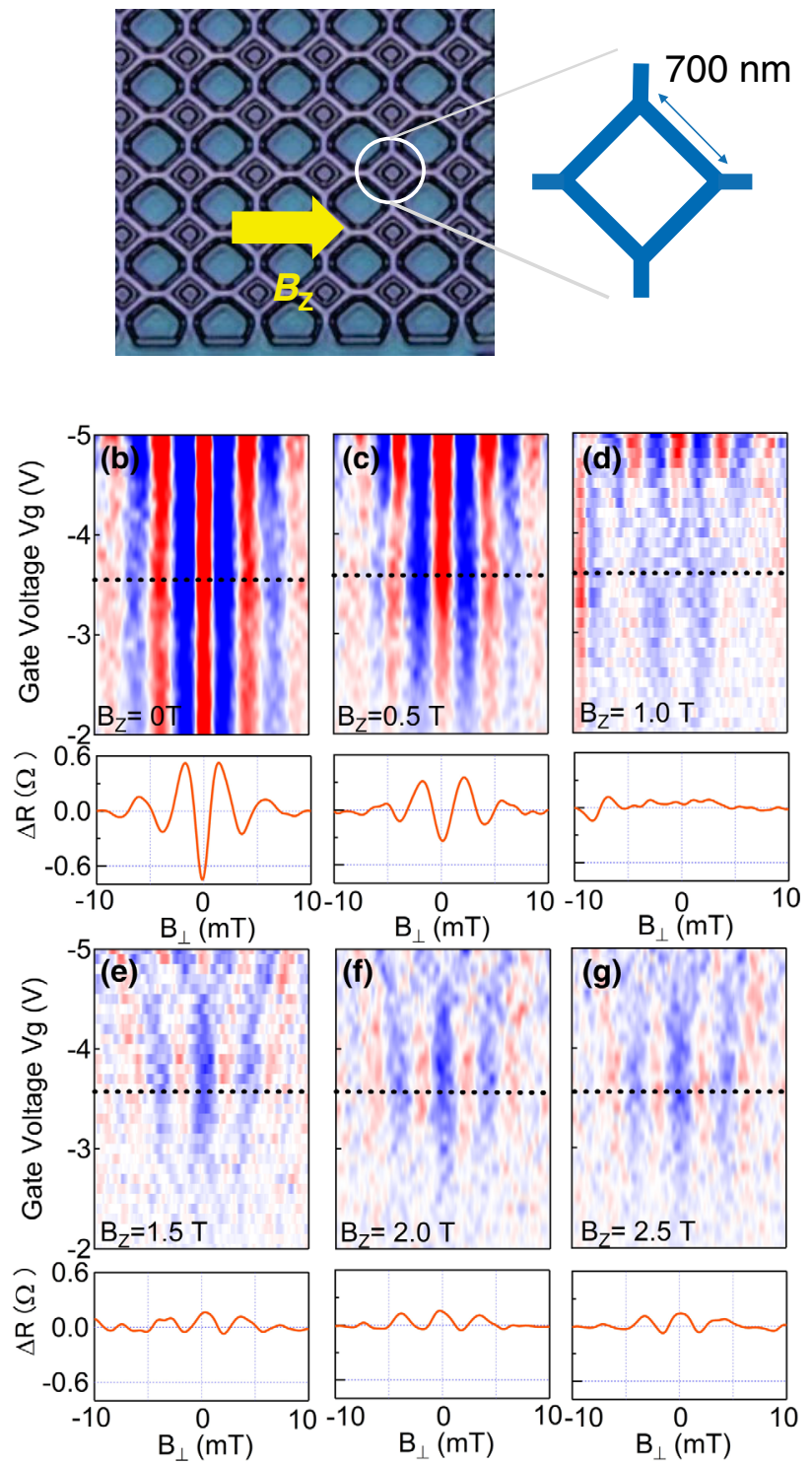

FIG. 3. (a) Laser scanning microscope image of a $40 \times 40$ square loop array with square side length $l=700 \mathrm{~nm}$. (b)-(g) Contour color plots of MR as a function of $V_{g}$ at different in-plane field strengths $B_{Z}$. The insets of (b)-(g) contain the MR data at $V_{g}=-3.6 \mathrm{~V}$, showing the AAS oscillations.

were performed at a temperature of $1.7 \mathrm{~K}$. The in-plane magnetic field $B_{Z}$ points along the squares' diagonal.

We focus our study on the $B_{Z}$ dependence of the Al'tshuler-Aronov-Spivak (AAS) [34] oscillation amplitude due to TR-path interference in the presence of a magnetic flux produced by a perpendicular field $B_{\perp}$. The AAS amplitude at $B_{\perp}=0$ as a function of top-gate voltages reflects exclusively the phase contribution from the spin part of the wave function. The sign reversal of the AAS oscillations indicates that the constructive spin interference switches to destructive interference (WAL), or vice versa (WL restoration).
We measured the magnetoresistance (MR) by applying a weak $B_{\perp}$ (few $\mathrm{mT}$ strength) as a function of the gate voltage $V_{g}$ that controls $\alpha$. A series of MR measurements was performed for different values of $B_{Z}$, running from $B_{Z}=0$ to $B_{Z}=2.5 \mathrm{~T}$ in steps of $\Delta B_{Z}=0.5 \mathrm{~T}$. Figures 3(b) $-3(\mathrm{~g})$ show the contour color plots of the MR as a function of $V_{g}$ for different $B_{Z}$. The insets in Figs. 3(b)-3(g) contain MR data at $V_{g}=-3.6 \mathrm{~V}$, showing AAS oscillations with period $\Delta B_{\perp}=(h / 2 e) l^{2}$ due to TR-path interference. The MR amplitude at $B_{\perp}=0$ as a function of $V_{g}$ corresponds to the AC spin interference [35,36] (definite AC modulations by $V_{g}$ are better seen in Figs. S4 and S5 [24]). As discussed in Fig. 1(b), the AC oscillation period in the square loop is 2 times larger than the $\mathrm{AC}$ period of the ring-shaped spin interferometer. This is the reason why a whole period of the $\mathrm{AC}$ oscillation is not observed in the present sample $[-3.62<\alpha<-1.17 \mathrm{peV} \mathrm{m}$, corresponding to a 1.5 window in $k_{\mathrm{SO}} P(\pi)$, Fig. 1(b)].

By increasing $B_{Z}$, the interference amplitudes in Figs. 3(b) $-3(\mathrm{~g})$ weaken gradually due to spin-induced dephasing [18] all over the gate voltage range. Still, a definite structure emerges as a function of $B_{Z}$. Around $B_{Z}=1 \mathrm{~T}$, the AAS amplitude vanishes at $V_{g}=-3.6 \mathrm{~V}$. Further increase in $B_{Z}(>1.5 \mathrm{~T})$ turns the AAS oscillations on again, but with a reversed AAS interference pattern. The sign reversal of the AAS oscillations corresponds to the transition from a destructive spin interference (WAL) to a constructive one (WL). This is clearly seen in Fig. 4(a). It is interesting to compare this response with the corresponding one observed in a ring array, shown in Fig. 4(b) for a fixed $V_{g}=-4.6 \mathrm{~V}$ (corresponding to $\alpha=-2.9 \mathrm{peV} \mathrm{m}$ ). The sample (ring radius $600 \mathrm{~nm}$ ) is the same one used in Ref. [4]. No inversion of the AAS oscillation pattern as a
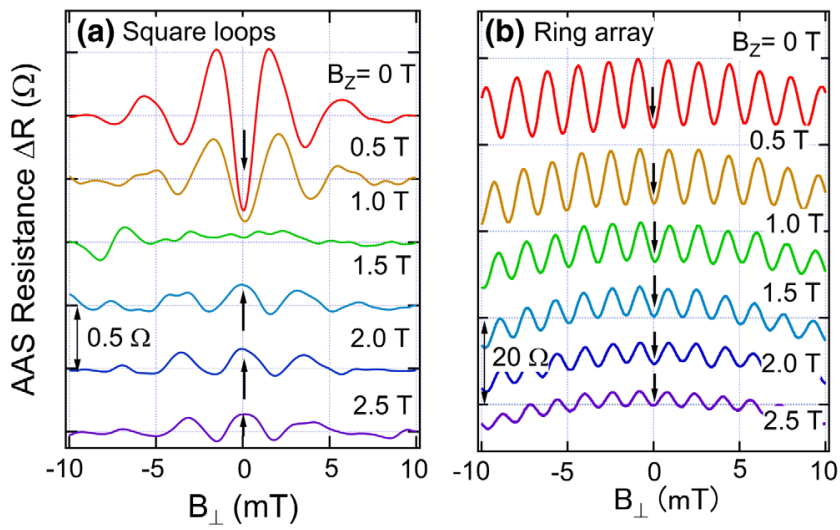

FIG. 4. (a) AAS oscillations for a square loop array at $V_{g}=$ $-3.6 V(\alpha=-2.5 \mathrm{peV} \mathrm{m})$ with different in-plane Zeeman fields $B_{Z}$. The sign reversal of the AAS oscillations corresponds to the transition from a destructive spin interference (WAL) to a constructive one (WL). (b) AAS oscillations for a ring array with radius $r=600 \mathrm{~nm}$ at $\alpha=-2.9 \mathrm{peV} \mathrm{m}$. The transition from WAL to WL is not observed in the ring array. 
function of $B_{Z}$ is observed in this case, so that the topological transition from WAL to WL never occurs in the studied gate voltage region. These results are consistent with the calculations shown in Figs. 1(a) and 1(b) (striped vs checkerboard patterns) based on the 1D model. The observed AAS oscillation reversal in the square loop array is attributed to the topological transition in the spin textures as discussed in Figs. 1 and 2.

In a previous work, we demonstrated [17] that the topological transition in a ring happens when the in-plane Zeeman field equals the Rashba SO field (namely, a topological transition in the spin texture requires a topological transition in field texture), which is given by $B_{\text {SO }}=$ $2 \alpha k_{F} / g \mu_{B}$. The corresponding in-plane Zeeman field is estimated to be $B_{Z}=6.7 \mathrm{~T}$ with $\alpha=-2.5 \mathrm{peV} \mathrm{m}$, $N_{s}=1.6 \times 10^{12} \mathrm{~cm}^{-2}$, and $g=4$. The experimentally observed transition field in the square loop array is around $B_{Z}=1.5 \mathrm{~T}$, which is much weaker than the value expected in the ring-shaped interferometer and does not require a topological change in the hybrid RashbaZeeman field texture [see points (B) and (D) in Figs. 1 and 2 for illustration]. This is the main result of our work.

Two-dimensional (2D) transport simulations.-The 1D model is useful to gain physical insight into the role played by the shape of the SO interference loop. However, it does not clarify how the topological transition is affected by scattering and multimode transport. Therefore, we resort to fully quantum 2D numerical simulations of disordered multi-mode square loops accounting for more realistic conditions. We implement a tight-binding model that is solved by using the Kwant code [37] with parameters fitting those in the InGaAs QW used in our experiment [24]. The numerical result shown in Fig. S6(a) of the Supplemental Material reproduces the experimental findings of Fig. 4(a) rather well. We further notice that the critical $B_{Z}$ producing the topological transition depends on the mean free path as shown in Fig. S6(b) [24]. This finding is compatible with previous results showing that disorder contributes to the development of nonadiabatic spin dynamics [6], boosting the effects of SO-field discontinuities at the square's corners.

This work was supported by the Japan Society for the Promotion of Science through Grant-in-Aid for Specially Promoted Research No. 15H05699, Grantin-Aid for Scientific Research (C) No. 17K05510, Grant-in-Aid for Innovative Areas No. JP15K21717, and Core-to-Core Program, and by Projects No. FIS201453385-P and No. FIS2017-86478-P (MINECO/FEDER, Spain). D. F. acknowledges additional support from the Marie Sklodowska-Curie Grant Agreement No. 754340 (EU/H2020). The 2D simulations were calculated using the HOKUSAI system provided by Advanced Center for Computing and Communication (ACCC) at RIKEN. J. N. and M.W. are grateful to Makoto Kohda for valuable discussions. *frustaglia@us.es

†nitta@material.tohoku.ac.jp

[1] A. Manchon, H. C. Koo, J. Nitta, S. M. Frolov, and R. A. Duine, New perspectives for Rashba spin-orbit coupling, Nat. Mater. 14, 871 (2015).

[2] Y. Aharonov and A. Casher, Topological Quantum Effects for Neutral Particles, Phys. Rev. Lett. 53, 319 (1984).

[3] Y. Aharonov and D. Bohm, Significance of electromagnetic potentials in the quantum theory, Phys. Rev. 115, 485 (1959).

[4] F. Nagasawa, D. Frustaglia, H. Saarikoski, K. Richter, and J. Nitta, Control of the spin geometric phase in semiconductor quantum rings, Nat. Commun. 4, 2526 (2013).

[5] Y. Lyanda-Geller, Topological Transitions in Berry's Phase Interference Effects, Phys. Rev. Lett. 71, 657 (1993).

[6] M. Popp, D. Frustaglia, and K. Richter, Conditions for adiabatic spin transport in disordered systems, Phys. Rev. B 68, 041303(R) (2003).

[7] M. V. Berry, Quantal phase factors accompanying adiabatic changes, Proc. R. Soc. A 392, 45 (1984).

[8] D. Loss, P. Goldbart, and A. V. Balatsky, Berry's Phase and Persistent Charge and Spin Currents in Textured Mesoscopic Rings, Phys. Rev. Lett. 65, 1655 (1990).

[9] A. G. Aronov and Y. B. Lyanda-Geller, Spin-Orbit Berry Phase in Conducting Rings, Phys. Rev. Lett. 70, 343 (1993).

[10] A. Stern, Berry Phase, Motive Forces, and Mesoscopic Conductivity, Phys. Rev. Lett. 68, 1022 (1992).

[11] T.-Z. Qian and Z.-B. Su, Spin-Orbit Interaction and Aharonov-Anandan Phase in Mesoscopic Rings, Phys. Rev. Lett. 72, 2311 (1994).

[12] A. F. Morpurgo, J. P. Heida, T. M. Klapwijk, B. J. van Wees, and G. Borghs, Ensemble-Average Spectrum of AharonovBohm Conductance Oscillations: Evidence for Spin-Orbit Induced Berry's Phase, Phys. Rev. Lett. 80, 1050 (1998).

[13] J.-B. Yau, E. P. De Poortere, and M. Shayegan, AharonovBohm Oscillations with Spin: Evidence for Berry's Phase, Phys. Rev. Lett. 88, 146801 (2002).

[14] F. E. Meijer, A. F. Morpurgo, and T. M. Klapwijk, One dimensional ring in the presence of Rashba spin-orbit interaction: Derivation of the correct Hamiltonian, Phys. Rev. B 66, 033107 (2002).

[15] M. Hentschel, H. Schomerus, D. Frustaglia, and K. Richter, Aharonov-Bohm physics with spin. Geometric phases in one-dimensional ballistic rings, Phys. Rev. B 69, 155326 (2004).

[16] F. Ghahari et al., An on/off Berry switch in circular graphene resonators, Science 356, 845 (2017).

[17] H. Saarikoski, J. E. Vázquez-Lozano, J. P. Baltanás, F. Nagasawa, J. Nitta, and D. Frustaglia, Topological transitions in spin interferometers, Phys. Rev. B 91, 241406(R) (2015).

[18] F. E. Meijer, A.F. Morpurgo, T. M. Klapwijk, and J. Nitta, Universal Spin-Induced Time Reversal Symmetry Breaking in Two-Dimensional Electron Gases with Rashba Spin-Orbit Interaction, Phys. Rev. Lett. 94, 186805 (2005).

[19] Z.-J. Ying, P. Gentile, C. Ortix, and M. Cuoco, Designing electron spin textures and spin interferometers by shape deformations, Phys. Rev. B 94, 081406(R) (2016). 
[20] Z.-J. Ying, P. Gentile, J. P. Baltanás, D. Frustaglia, C. Ortix, and M. Cuoco, Geometric driving of two-level quantum systems, arXiv:1909.04291.

[21] D. Bercioux, D. Frustaglia, and M. Governale, Signatures of spin-related phases in transport through regular polygons, Phys. Rev. B 72, 113310 (2005).

[22] M. J. van Veenhuizen, T. Koga, and J. Nitta, Spin-orbit induced interference of ballistic electrons in polygon structures, Phys. Rev. B 73, 235315 (2006).

[23] The value of $N$ necessary for the good description of a Rashba ring must be determined by setting the side length $L$ much smaller than the spin precession length $L_{\mathrm{SO}}=\pi / k_{\mathrm{SO}}$.

[24] See Supplemental Material at http://link.aps.org/ supplemental/10.1103/PhysRevLett.123.266804 for technical summaries and extended discussions on semiclassical theory of conductance, AC and AAS oscillations in 1D ring and square loops, layer structure of the InGaAs quantum well, AC spin interference in experiments with square loops, and 2D numerical simulations of transport, which includes Refs. [25-28].

[25] K. Richter, Semiclassical Theory of Mesoscopic Quantum Systems (Springer, Berlin, 2000); R. A. Jalabert, in New Directions in Quantum Chaos, edited by G. Casati, I. Guarneri, and U. Smilansky (IOS Press, Amsterdam, 2000).

[26] H. Frisk and T. Guhr, Spin-orbit coupling in semiclassical approximation, Ann. Phys. (N.Y.) 221, 229 (1993).

[27] D. Loss and P. M. Goldbart, Persistent currents from Berry's phase in mesoscopic systems, Phys. Rev. B 45, 13544 (1992).

[28] D. Frustaglia, Spin-dependent transport in mesoscopic quantum systems, Ph.D. thesis, Technische Universität Dresden, 2001.

[29] Formal problems in the definition of $\omega_{s / B}$ for squares are eliminated by introducing rounded corners with radius $r \ll L$ that remove any discontinuity in the field texture and in the spin texture's derivative without other significant consequences. Otherwise, notice that $\omega_{s}$ can always be calculated by piecewise integration along the square's sides.

[30] D. Frustaglia and K. Richter, Spin interference effects in ring conductors subject to Rashba coupling, Phys. Rev. B 69, 235310 (2004).

[31] A. A. Reynoso, J. P. Baltanás, H. Saarikoski, J. E. VázquezLozano, J. Nitta, and D. Frustaglia, Spin resonance under topological driving fields, New J. Phys. 19, 063010 (2017).

[32] J. Nitta, T. Akazaki, H. Takayanagi, and T. Enoki, Gate Control of Spin-Orbit Interaction in an Inverted InGaAs/InAlAs Heterostructure, Phys. Rev. Lett. 78, 1335 (1997).

[33] G. Engels, J. Lange, Th. Schäpers, and H. Lüth, Experimental and theoretical approach to spin splitting in modulation-doped InGaAs/InP quantum wells for $B \rightarrow 0$, Phys. Rev. B 55, R1958(R) (1997).

[34] B. L. Al'tshuler, A. G. Aronov, and B.Z. Spivak, The Aharonov-Bohm effect in disordered conductors, JETP Lett. 33, 94 (1981), http://www.jetpletters.ac.ru/ps/1501/ article_22943.shtml.

[35] T. Bergsten, T. Kobayashi, Y. Sekine, and J. Nitta, Experimental Demonstration of the Time Reversal AharonovCasher Effect, Phys. Rev. Lett. 97, 196803 (2006).

[36] F. Nagasawa, J. Takagi, Y. Kunihashi, M. Kohda, and J. Nitta, Experimental Demonstration of Spin Geometric Phase: Radius Dependence of Time-Reversal AharonovCasher Oscillations, Phys. Rev. Lett. 108, 086801 (2012).

[37] C. W. Groth, M. Wimmer, A. R. Akhmerov, and X. Waintal, Kwant: A software package for quantum transport, New J. Phys. 16, 063065 (2014). 